\title{
The Geometric Algebra Lift of Qubits and Beyond
}

\author{
Alexander Soiguine ${ }^{1}$ \\ ${ }^{1}$ SOiGUINE Quantum Computing
}

January 7, 2022

\begin{abstract}
The Geometric Algebra formalism opens the door to developing a theory upgrading conventional quantum mechanics. Generalizations, stemming from implementation of complex numbers as geometrically feasible objects in three dimensions; unambiguous definition of states, observables, measurements bring into reality clear explanations of conventional weird quantum mechanical features, particularly the results of double split experiments where particles create diffraction patterns inherent to wave diffraction. This weirdness of the double split experiment is milestone of all further difficulties in interpretation of quantum mechanics.
\end{abstract}

\section{Hosted file}

Geometric Algebra Lift of Qubits and Beyond.docx available at https://authorea.com/users/ 454445/articles/552055-the-geometric-algebra-lift-of-qubits-and-beyond 